En torno al
Pensamiento Franciscaco 

Página inicial: 317 - Página final: 333

Tipo de artículo: de investigación

\section{Percepciones declaradas por los estudiantes y profesores sobre las vivencias/experiencias de la misión y visión de la USB-Medellín. ${ }^{4}$}

Perceptions declared by the students and teachers on the experiences of the mission and vision of the USB-Medellin experiences.

Recibido: julio de 2015 Revisado: octubre de 2015

Aceptado: noviembre 20 de 2015

Por: Dora Inés Arroyave Giraldo', Verónica Moreno López ${ }^{2}$ y Luis Fernando Sánchez Sánchez ${ }^{3}$.

\section{Resumen.}

El siguiente texto presenta los resultados sobre el estudio, en torno a las percepciones declaradas por los estudiantes y profesores sobre las vivencia/experiencias de la misión y visión de la Universidad de San Buenaventura-Medellín (Colombia). La metodología utilizada fue mixta. El muestreo fue aleatorio estratificado. La técnica utilizada fue la encuesta, estructurada con 20 ítems, distribuidos en las tres categorias de análisis (Misión, Visión y Dimensiones de la Pedagogía Franciscana).

\section{Palabras clave.}

Misión, Visión, Pedagogía Franciscana, Vivencias, Experiencias, Estudiantes, Profesores, Identidad, Paideia, Universidad

\section{Abstract.}

The following text presents the results on the study, around perceptions declared by the students and professors about the ways of life and experiences of the mission and vision of Saint Bonaventure University, Medellin branch, Colombia. It used a mixed methodology. The sample was random, stratified. The technique used was the survey, which was structured with 20 items, distributed into three categories of analysis (Mission, Vision, and Dimensions of the Franciscan pedagogy).

\section{Key words.}

Mission; Vision; Franciscan pedagogy; Ways of life; Experiences; Students; Professors; Identity; Paideia; and University.

\footnotetext{
${ }^{1}$ Doctora en educación. Docente investigador. Línea Gestión Educativa-Currículo. Grupo ESINED. Facultad de Educación. Universidad de San Buenaventura. Contacto: dora.arroyave@usbmed.edu.co

${ }^{2}$ Magister en educación. Docente Investigadora. Línea Paideia. Grupo: GIDPAD. Unidad de Formación Humana. Universidad de San Buenaventura-Medellin. Contacto: veronica.moreno@usbmed.edu.co

${ }^{3}$ Magister en educación. licenciado Filosofia, especialista gestión Educativa. Docente Investigador, Línea Paideia. Grupo: GIDPAD. Unidad de Formación Humana. Universidad de San Buenaventura-Medellin. Contacto: Luis. Sanchez@usbmed.edu.co
} 


\section{Introducción.}

El Proyecto Educativo Bonaventuriano (2007, p. 68-69) propone la pedagogía franciscana como un proceso formativo, el cual se centra en la persona y se fundamenta en lo cotidiano, en las relaciones dialógicas fraternas y en la creatividad e imaginación.

Plantea entonces que "la pedagogía franciscana" abarca cuatro dimensiones, a saber:

- La persona: Es el centro de la pedagogía franciscana. No trata de la persona en genérico, ni de una teoría que defienda un concepto de hombre en particular, sino de la persona concreta que participa en el proceso formativo. En la pedagogía franciscana predomina el método intuitivo, donde la afectividad y el respeto a la espontaneidad de la persona son principios para la interacción humana y para los fines prácticos de la educación. En ella, se asume la singularidad como derecho inherente a la dimensión personal, de tal forma que respeta y no masifica los ritmos y estilos de aprendizaje del estudiante.

- Lo cotidiano: Es lo fundamental en la pedagogía franciscana. Recuperar lo cotidiano es posibilitar que la vida cargada de sentido y esperanza suceda en el escenario de lo pedagógico. La pedagogía franciscana no pretende formar para repetir o almacenar conceptos, sino para explorar las múltiples facetas de la persona como ser capaz de captar, dar y expresar sentido a la realidad mediante el diálogo consigo mismo, con el otro, con el entorno y con el trascendente.

- La relación dialógica fraterna: Tiene connotación significativa para propiciar el respeto, la tolerancia, la participación, el reconocimiento y la aceptación. Esta actitud pedagógica se concreta en la pedagogía de la fraternidad donde se concilian lo divino y lo humano fundamentados en el amor.

- Lo creativo: Es lo creativo, un continuo movimiento de búsqueda que genera ambientes propicios para la exploración y las posibilidades de imaginar, de crear y de encontrar formas diferentes para apropiarse del saber.

El documento "Propuesta de lineamientos curriculares del año 2013 de La U.S.B- Medellín, retoma el texto Id y Enseñad (Orden Franciscana Menor - Curia generale dei Frati Minori, 2009), el cual presenta siete realidades franciscanas enmarcadas en lo económico, político, ético, urbano, social, medio-ambiental y espiritual, es decir, aquellos asuntos definidos desde el franciscanismo, no sólo de interés mundial, que se deben tener en cuenta en un proceso de formación en cada una de las comunidades académicas franciscanas. Se propone interrelacionar esas siete realidades reflexivamente con las diferentes áreas disciplinares de la comunidad Universitaria Bonaventuriana pues, se articulan de manera intuitiva a éstas realidades:

- Realidad económica/Ingenierías y Ciencias empresariales, desde la reflexión sobre el hombre y la manera como produce tecnologías.

- Realidad social/Psicología y Educación: reflexión sobre el hombre y la manera como se comporta y relaciona construyendo sociedad.

- Realidad urbana/Arquitectura y Diseño: reflexión sobre el hombre en la construcción de ciudad.

- Realidad política/Derecho: reflexión sobre el como ser político que se relaciona a través de la norma. 
- Realidad ética/Formación Humana, hoy CIDEH (Centro interdisciplinario de estudios Humanísticos): reflexión sobre la construcción de valores, principios, integridad, cultura, identidad institucional e idoneidad profesional.

- Realidad medio ambiental/oportunidad para todas las disciplinas: reflexión sobre el entorno y la acción del hombre como ser globalizado, complejo y su responsabilidad ecológica

- Realidad espiritual/Pastoral universitaria y hoy, la recientemente instancia universitaria, denominada Dirección para la Evangelización de las culturas: reflexión sobre el hombre en su esencia misma y/o desde la persona de Jesucristo, la dimensión interior $\mathrm{y}$ espiritual, el hecho religioso del ser humano.

Desde esa lógica, se considera posible la aparición de nuevas realidades que permitirán generar procesos de transformación que, no han sido lo suficientemente exploradas desde las diferentes disciplinas, es decir, que no han integrado el problema del conocimiento, desde otras "aristas". (Arroyave y Carrión, 2013, p. 18)

\section{En cuanto a la percepción.}

Los autores del presente estudio distinguen el concepto de percepción siguiendo a autores como Borg, 1998; Castelló, 2001; quienes plantean que toda percepción comporta una interpretación, por lo que, más que tratarse de un fenómeno puramente sensorial, hay que considerarlo como una actividad compleja, vinculada a un cuadro particular de referencias elaborado a partir de la propia experiencia personal y social.

De otro lado, Pérez (1989) entiende la percepción como "una actividad que se estructura alrededor de los sentimientos, intenciones, motivaciones, valores, inserciones sociales y normativas que caracterizan al perceptor y al objeto percibido" (p.90).

Es por ello, que en el presente estudio se pretende caracterizar las percepciones declaradas por los estudiantes y profesores sobre las vivencias/experiencias de la Misión y Visión de la U.S.B-Medellín, abordando las perspectivas anteriormente planteadas: las cuatro dimensiones de la pedagogía y las siete realidades franciscanas.

Desde el anterior contexto se presentó la siguiente pregunta de Investigación:

¿Cuáles son las percepciones de estudiantes y profesores de la Universidad de San BuenaventuraMedellin sobre las vivencias/experiencias de la Misión y Visión Institucional?

Se propuso como objetivo: Caracterizar las percepciones declaradas por los estudiantes y profesores sobre las vivencias/experiencias de la Misión y Visión de la USB-Medellín.

\section{Metodologia.}

Por las características del estudio, se hizo indispensable la utilización de la metodología mixta (Hernández y otros, 2003) o multimétodo (Polit y Hungler, 2000), teniendo en cuenta ésta última referencia, se planteó un diseño integrado por componentes que incluyó aspectos tanto cuantitativos como cualitativos. 
La población objeto de estudio correspondió a los estudiantes que conformaban los grupos matriculados en los cursos transversales de Formación Humana, (hoy denominado C.I.D.E.H) y los profesores de pregrado de la Universidad de San Buenaventura-2014. El muestreo fue aleatorio estratificado, en ese sentido, la población total de estudiantes y profesores, se subdividió en poblaciones más pequeñas denominadas estratos: Estrato de estudiantes; 65.

Estrato de profesores: 55 .

La técnica utiliza fue la encuesta, estructurada con 20 ítems, distribuidos en las tres categorias de análisis (Misión, Visión y Dimensiones de la Pedagogía Franciscana), los cuales se presentaron en igual formato a los dos actores: estudiantes y profesores.

En el momento de análisis e interpretación se realizó un proceso de contrastación de resultados a través del hallazgo de coincidencias y contradicciones en las diversas respuestas declaradas por los estudiantes y profesores, a la vez que se interrelacionaron los datos con testimonios y pensamientos franciscanos.

\section{Resultados.}

Las percepciones declaradas por los estudiantes y profesores sobre las vivencias/ experiencias de la Misión y Visión de la U.S.B-Medellín, se analizaron desde tres categorias de análisis: Misión, Visión y Dimensiones de la Pedagogía Franciscana; a continuación la sintesis de los principales resultados:

\section{Desde la Misión.}

Frente a la pregunta: ¿Cuáles dimensiones de la misión universitaria son importantes para usted? Calificando de 1 a 5, donde 1 señala "No importa en lo más Mínimo" y 5 representa "Extremamente Importante" se encontró:

- En la dimensión denominada Ser Universitario: E1 0\% de Los docentes señalaron "no importa en lo más mínimo, el $2 \%$ "levemente importante"; como importante $38 \%$. Por parte de estudiantes, el $1 \%$ señaló "no importa en lo más mínimo", levemente el $1 \%$, importante el $18 \%$, muy importante $30 \%$.

En ambas poblaciones encuestadas, el porcentaje dado al item "extremadamente importante" es significativo al arrojar un $60 \%$ en docentes frente a un $49 \%$ en estudiantes.

- En la dimensión denominada Ser Franciscano: El 4\% de docentes señala "no importa en lo más mínimo"; 20\% "levemente importante", 35\% importante, extremadamente importante el 38\%. Por parte de los discentes, no importa en lo más mínimo 7\%, levemente importante $15 \%$, importante $39 \%$, muy importante $19 \%$, extremadamente importante $19 \%$.

- En la dimensión denominada Ser católico: 16\% de docentes señalan "no importa en lo más mínimo", "levemente importante" $18 \%$, importante $38 \%$, extremadamente importante $27 \%$. Los estudiantes señalaron el ítem "no importa en lo más mínimo" en un 16\%, levemente importante $16 \%$, importante $27 \%$, extremadamente importante $21 \%$. 
Sorprende el observar que, frente al carácter católico en ambos grupos, calificaron la opción "extremadamente importante" con un porcentaje bajo: docentes $27 \%$ y estudiantes $21 \%$, representando éste resultado aproximadamente una quinta parte de ambos grupos. Frente al ser franciscano se encontró que un $38 \%$ de los docentes afirman extremadamente importante, frente a un $19 \%$ de los estudiantes.

Respecto a coincidencias, se encontró que ambos grupos, señalaron en un 16\% a la opción "No importa en lo más mínimo" al ítem "dimensión católica". Ambos grupos señalaron en un $7 \%$ el ítem "No importa en los más mínimo", en el Ser franciscano.

Resulta necesario entonces recordar que, como tendencia educativa, desde el pensamiento franciscano se plantea que:

El discurso franciscano, desde su dimensión educativa y formativa ocupa un lugar dentro del Campo Conceptual de la Pedagogía al inscribirse en la dinámica de tener discursos que la constituyen, prácticas que sustenten o detenten el discurso educativo franciscano e instituciones que regulen, normaticen y dinamicen las prácticas y los discursos. (Paideia franciscana, 2006, p. 44)

En cuanto a la manera como reflejan en el actuar, tanto profesores como estudiantes bonaventurianos, las dimensiones de la misión universitaria en su entorno; en las respuestas dadas por los estratos encuestados se encontró que:

\section{Tabla 1: Manera como reflejan en el actuar, tanto profesores como estudiantes bonaventurianos, las dimensiones de la misión universitaria en su entorno. Percepciones.}

\begin{tabular}{|c|c|}
\hline Items & $\begin{array}{ll}\text { Valoración - resultados } \\
\end{array}$ \\
\hline $\begin{array}{l}\text { Frente al trabajo en equipo } \\
\text { como elemento axiológico } \\
\text { actitudinal de lo misional. }\end{array}$ & $\begin{array}{l}\text { El 56\% de los docentes considera que éste ítem, lo refleja en su actuar } \\
\text { "siempre", "casi siempre" } 31 \% \text {, "algunas veces" } 13 \% \text {, "casi nunca" 0\%, } \\
\text { "nunca" 0\%. Respecto al item, los estudiantes señalaron "siempre" } 49 \% \text {, } \\
\text { "casi siempre" } 34 \% \text {, "algunas veces" } 13 \% \text {, "casi nunca y nunca" } 0 \% \text {. Resulta } \\
\text { altamente significativo que la población docente en un } 56 \% \text { manifieste su } \\
\text { prioridad y cuidado en la relación de su saber específico con las dimensiones } \\
\text { de su misión y visión, y que de forma simultánea, los discentes participantes } \\
\text { en la encuesta, manifiesten encontrar ésta intencionalidad en sus contextos } \\
\text { formativos y espacios curriculares en un 34\%. }\end{array}$ \\
\hline $\begin{array}{l}\text { En relación a la actitud de } \\
\text { servicio. }\end{array}$ & $\begin{array}{l}\text { Se observa que el } 75 \% \text { de los docentes encuestados expresa que busca } \\
\text { reflejar ésta, en su comportamiento; en los estudiantes corresponde esta } \\
\text { misma respuesta a un } 54 \% \text {. }\end{array}$ \\
\hline $\begin{array}{l}\text { En cuanto al saber } \\
\text { disciplinar. }\end{array}$ & $\begin{array}{l}\text { El } 56 \% \text { de los docentes participantes afirman que lo reflejan "siempre", al } \\
\text { igual que el } 46 \% \text { de los estudiantes. }\end{array}$ \\
\hline $\begin{array}{l}\text { En calidad humana } y \\
\text { profesional: }\end{array}$ & $\begin{array}{l}\text { El } 80 \% \text { de los docentes respondió que "siempre" lo han tenido en cuenta en } \\
\text { relación a un } 64 \% \text { de los estudiantes. }\end{array}$ \\
\hline En el comportamiento ético & $\begin{array}{l}\text { E1 } 85 \% \text { de los docentes manifiestan que "siempre" buscan reflejarlo en sus } \\
\text { acciones, frente a un } 57 \% \text { del estudiantado. }\end{array}$ \\
\hline $\begin{array}{l}\text { frente al respeto por la } \\
\text { diferencia }\end{array}$ & $\begin{array}{l}\text { El } 84 \% \text { de los docentes afirma que lo refleja en sus comportamientos y } \\
\text { decisiones frente al } 63 \% \text { de los estudiantes. }\end{array}$ \\
\hline
\end{tabular}

Fuente: propia 
Llama la atención que en el caso de los docentes, sea el comportamiento ético, muy cerca al respeto por la diferencia el ítem evaluado con el mayor puntaje. En el caso de los estudiantes el mayor puntaje obtenido es por el concepto de calidad humana y profesional, seguido muy de cerca por el respeto por la diferencia.

Ahora bien, en el elemento axiológico y actitudinal de trabajo en equipo, coinciden tanto docentes como estudiantes encuestados en señalar que lo reflejan "algunas veces" solo en un $13 \%$. En cuanto a la resolución de problemas, el $36 \%$ coincidieron en señalar que "casi siempre" lo hacen. Y referente a la actitud de servicio, respeto a la diferencia, calidad humana y profesional, tanto los docentes como estudiantes coincidieron en decir que en un $0 \%$ dejan de reflejar la actitud de servicio en sus actos y decisiones.

Para el aspecto relacionado con el reflejo de las dimensiones de la misión universitaria en las diversas actuaciones de los actores: estudiantes/profesores, se encuentra relevante explicitar lo que señala el documento Id y enseñad en cuanto a que:

La crisis ética, cultural, existencial y económica que padece la sociedad, por consiguiente, no encontrará solución en las ofertas técnicas y económicas, sino en un cambio profundo de actitudes. Es aquí en donde la educación franciscana puede y debe proponer como alternativa antropológica un modelo de persona que sea abierta a lo trascendente y portadora de una dignidad que la constituye en un absoluto frente a los objetos y que, por lo mismo, no se puede manipular, objetivar ni engañar. (Orden Franciscana Menor - Curia generale dei Frati Minori, 2009)

En relación a la importancia que en las diversas asignaturas/cursos/seminarios que se desarrollan en el proceso de formación profesional se considere fundamental la búsqueda constante de la verdad, el análisis serio y objetivo de la realidad, el rigor científico intrínseco de la ciencia, el valor de la investigación y el examen crítico de los conocimientos y su aplicación al desarrollo de la comunidad; la población encuestada declaró en:

Tabla 2: Nivel de Importancia que le otorgan los encuestados a cadauno de los items señalados en la encuesta dentro de las asignaturas/cursos/seminarios

\begin{tabular}{|l|l|}
\hline Ítem & Valoración - resultados \\
\hline La verdad. & $\begin{array}{l}\text { El 45\% de docentes manifiesta que es "extremadamente importante" } \\
\text { la búsqueda y conocimiento de la verdad en sus prácticas, en } \\
\text { relación a un 57\% de los estudiantes, que afirman igualmente } \\
\text { considerarlo "extremadamente importante". }\end{array}$ \\
\hline Análisis objetivo de la realidad. & $\begin{array}{l}\text { El 55\% de los docentes dicen que esto es "extremadamente } \\
\text { importante" en relación a un 54\% de los estudiantes; un margen } \\
\text { minimo de diferencia. }\end{array}$ \\
\hline $\begin{array}{l}\text { Rigor cientifico intrínseco de la } \\
\text { ciencia. }\end{array}$ & $\begin{array}{l}\text { El33\% de los docentes señalaron que lo consideran "extremadamente } \\
\text { importante", a la par que el 42\% de los dicentes señalan el mismo } \\
\text { item. }\end{array}$ \\
\hline El valor de la investigación. & $\begin{array}{l}\text { Como "extremadamente importante" los docentes lo señalan en 53\% } \\
\text { y estudiantes en un 39\%. }\end{array}$ \\
\hline Examen crítico del conocimiento: & $\begin{array}{l}\text { Como "extremadamente importante" señala el 58\% de docentes y el } \\
46 \% \text { de estudiantes. }\end{array}$ \\
\hline
\end{tabular}

Fuente: propia 
Los elementos evaluados con los mayores porcentajes, son: en el caso de los docentes el examen crítico de los conocimientos y su aplicación al desarrollo de la comunidad. En el caso de los estudiantes, es la búsqueda constante de la verdad.

De otro lado, en el rigor cientifico intrínseco de la ciencia; en este aspecto coinciden ambos grupos en un 16\%, al afirmar que es "importante". Y en el valor de la investigación: ambos grupos encuestados coincidieron en señalar que este aspecto es "importante" en un $40 \%$.

En ese sentido, encontramos pertinente reafirmar la misión institucional en el marco del pensamiento franciscano desde diversos textos:

Desde el texto de La educación franciscana como un agente de cambio, cuando sentencia que:

...la educación franciscana es caracterizada como un matrimonio del intelecto y el afecto, una unión de la cabeza y el corazón... (Perry, 2013, p. 20). La tarea de la educación es formar y trasformar a sus estudiantes intelectual, afectiva y conductualmente, y a través de ellos y con ellos trasformar el mundo. (Perry, 2013, p. 16).

Desde el Proyecto Educativo Bonaventuriano, en tanto propone que el estudiante:

- Asume proactivamente su formación académica y su formación humana;

- Se forma como persona digna y profesional, con criterios, competencias y conocimientos suficientes para identificar diferentes problemáticas y liderar sus soluciones.

- Enfrenta y cuestiona las realidades humanas y de su disciplina con enfoques transdisciplinarios;

- Se reconoce como ser histórico e integral que se construyen el desarrollo de sus contradicciones y en las interacciones con sus semejantes. (PEB, 2007, p. 53).

En cuanto al docente, se específica que:

El docente bonaventuriano practica la pedagogía franciscana que lo compromete a ser: un facilitador del aprendizaje, capaz de diseñar y desarrollar estrategias metodológicas que respondan a las caracteristicas de los saberes y de las personas que los reciben. (PEB, 2007, p. 52).

Finalmente, desde el documento Id y enseñad, señala que:

Una educación, por tanto, que enseñe a leer y a escribir la realidad, a interpretarla y actuar sobre ella, con un espíritu crítico - constructivo. (OFM, 2009, p. 15).

En sintesis, puede caracterizarse la misión institucional, en términos de fortalezas y debilidades en el siguiente cuadro: 


\begin{tabular}{|c|c|c|c|}
\hline \multicolumn{4}{|c|}{ CATEGORÍA MISIÓN } \\
\hline \multicolumn{2}{|c|}{ FORTALEZAS } & \multicolumn{2}{|c|}{ DEBILIDADES } \\
\hline PROFESORES & ESTUDIANTES & ROFESORES & ESTUDIANTES \\
\hline $\begin{array}{l}\text { Se afirma de parte de los } \\
\text { docentes, que su actuar en el } \\
\text { ejercicio de su labor, } \\
\text { corresponde con la misión de la } \\
\text { Universidad. Existe } \\
\text { identificación con los principios } \\
\text { que la componen y con la } \\
\text { vivencia de los mismos en las } \\
\text { actividades cotidianas. } \\
\text { La investigación demostró un } \\
\text { nivel de apropiación de la } \\
\text { misión institucional desde las } \\
\text { prácticas curriculares. } \\
\text { Los datos y hallazgos } \\
\text { estadísticos sobre la misión, } \\
\text { muestran que no existe una } \\
\text { brecha considerable entre la } \\
\text { percepción de los docentes y } \\
\text { las de los estudiantes frente al } \\
\text { tema identitario. } \\
\text { El ser franciscano, como eje } \\
\text { protagónico de la filosofía }\end{array}$ & $\begin{array}{l}\text { En general se reconoce la } \\
\text { misión como parte } \\
\text { fundamental de la actividad } \\
\text { cotidiana de la Universidad y } \\
\text { de sus estudiantes. Ésta es } \\
\text { bien vista y aceptada de } \\
\text { manera amplia. } \\
\text { La investigación dejó observar } \\
\text { un conocimiento formal y } \\
\text { enunciativo de la misión en } \\
\text { los estudiantes. } \\
\text { Hay una confirmación de los } \\
\text { postulados del pensamiento } \\
\text { franciscano en el promedio de } \\
\text { ambas poblaciones } \\
\text { consultadas. } \\
\text { El eje misional denominado } \\
\text { "ser católico", es una } \\
\text { dimensión entendida y } \\
\text { aceptada por el estudiante } \\
\text { como parte constitutiva de la } \\
\text { institución universitaria que }\end{array}$ & $\begin{array}{l}\text { Es importante que el docente } \\
\text { se acerque a los principios } \\
\text { franciscanos, pues pareciera } \\
\text { ser que a veces se identifican } \\
\text { estos más como elementos } \\
\text { impuestos que como } \\
\text { propuesta de vida aplicable a lo } \\
\text { cotidiano. Se percibe que la } \\
\text { dimensión católica y la } \\
\text { franciscana, no tienen tanto } \\
\text { peso en términos de } \\
\text { importancia dentro de la } \\
\text { misión. }\end{array}$ & $\begin{array}{l}\text { Se perciben ciertas carencias } \\
\text { en la transmisión de los } \\
\text { fundamentos y elementos de } \\
\text { la misión institucional a los } \\
\text { estudiantes; cuando estos se } \\
\text { mencionan de manera } \\
\text { explícita, los estudiantes } \\
\text { pueden establecer relaciones } \\
\text { con sus actividades cotidianas, } \\
\text { pero no los identifican con } \\
\text { claridad por sí mismos. } \\
\text { Llama la atención que siempre } \\
\text { al mencionar el aspecto } \\
\text { franciscano o católico, los } \\
\text { estudiantes parecieran } \\
\text { relacionarlo con } \\
\text { adoctrinamiento, un } \\
\text { comprender que la axiología } \\
\text { que ello trae, hace parte del } \\
\text { proceso académico cotidiano. } \\
\text { Hay una percepción de tener } \\
\text { claridad en la formulación } \\
\text { formal de la Misión, de los ejes }\end{array}$ \\
\hline
\end{tabular}

Cuadro 1. Fortalezas y debilidades de la categoría Misión

\section{Desde la Visión.}

Respecto a la pregunta relacionada con la importancia dada a los componentes de la Visión institucional, se observó una mínima diferencia en ambos grupos encuestados al señalar como "extremadamente importante" el $62 \%$ de los docentes y el $63 \%$ de los estudiantes, lo concerniente con la innovación pedagógica y el fomento de la sencillez en las relaciones. En la producción investigativa; para el 60\% de los estudiantes es "extremadamente importante", mientras que para los profesores arroja el 49\%. En la calidad cientifica; el 61\% de estudiantes lo señala como "extremadamente importante" frente al $40 \%$ de los docentes. En impacto y transformación social; el $73 \%$ de la población docente afirma que es "extremadamente importante" mientras que un 63\% de la población estudiantil eligió el mismo ítem.

Así las cosas, se evidencia como el concepto de impacto y transformación social tiene el más alto porcentaje de calificación siendo "extremadamente importante" tanto para estudiantes como para docentes. De hecho en los testimonios se corrobora tal situación,asi:

Testimonio de profesor, el cual afirma, entre otros lo siguiente; "Señalando con distintos ejemplos reales los posibles impactos de la actuación profesional en la ciudad sobre las oportunidades y vivencias de grupos sociales de menores recursos".

"En la solidaridad y la ayuda hacia las demás personas que más lo necesitan". Testimonio de estudiante. 
Lo anterior genera un reto enorme para la educación franciscana, en tanto desde sus directrices se propone que:

Es una certeza que estamos viviendo no solamente una época de cambios, sino un cambio de época, marcada por otros paradigmas o modelos de persona, de familia y de sociedad y, en consecuencia, de sistemas educativos. (OFM, 2009, p. 10).

Y como desafío nos plantea que:

...primer desafio para la educación franciscana: ¿Cómo proponer, con claridad, creatividad y audacia, a las generaciones actuales, un nuevo paradigma de relación con la naturaleza, con los hombres, con Dios y consigo mismo? (2009, p. 10).

También desde el texto de La educación franciscana como agente de cambio, se invita a comprender el proceso formativo desde la siguiente perspectiva: "A cualquier nivel en cualquier escenario la educación se refiere fundamentalmente a la transformación humana" (Perry, 2013, p. 16).

Por medio de la tabla 3, a continuación presentada, el lector puede identificar lo relacionado con los procesos de formación, a través de las diversas asignaturas/cursos/seminarios y por tanto lo observado por el equipo investigador:

Tabla 3. Los docentes consideran los procesos de formación que se desarrollan en la Universidad, a través de las diversas asignaturas/cursos/seminarios, contribuyen asi:

\begin{tabular}{|c|c|}
\hline Valoración & Ítem --------Resultado \\
\hline "Siempre" & $\begin{array}{l}\text { En un } 24 \% \text { a la formación patriótica, } \\
\text { En un } 31 \% \text { a la formación espiritual, } \\
\text { En un } 42 \% \text { a la formación tecnológica, } \\
\text { En un } 44 \% \text { a la formación ecológica, } \\
\text { En un } 45 \% \text { a lo cultural } \\
\text { En un } 45 \% \text { a la formación científica, } \\
\text { En un } 60 \% \text { a la formación social, } \\
\text { En un } 76 \% \text { a lo ético. }\end{array}$ \\
\hline "Casi Siempre" & $\begin{array}{l}\text { En un } 20 \% \text { a lo ético. } \\
\text { En un } 31 \% \text { a la formación social, } \\
\text { En un } 36 \% \text { a la formación científica, } \\
\text { En un } 36 \% \text { a la formación ecológica, } \\
\text { En un } 36 \% \text { a la formación tecnológica, } \\
\text { En un } 40 \% \text { a la formación patriótica, } \\
\text { En un } 42 \% \text { a la formación espiritual, } \\
\text { En un } 47 \% \text { a lo cultural }\end{array}$ \\
\hline
\end{tabular}

Fuente: propia 
Llama la atención como el ítem que más se destaca en el proceso es el de formación ética, respondiendo a un $76 \%$ bajo la premisa de estar siempre presente en los procesos formativos. Por su parte el item con menos incidencia porcentual, bajo la respuesta “ siempre" es el de Formación Patriótica. También se destaca la formación de tipo espiritual, con una participación menor en relación a los demás ítems.

En el rol de estudiante, las respuestas generadas se describen así;

Tabla 4. Los Estudiantes consideran que los procesos de formación a través de las diversas asignaturas/cursos/seminarios que reciben en la Universidad, contribuyen asi :

\begin{tabular}{|c|c|}
\hline Valoración & Items - resultados \\
\hline "Siempre" & $\begin{array}{l}\text { En un } 25 \% \text { a la formación patriótica, } \\
\text { En un } 30 \% \text { a la formación espiritual, } \\
\text { En un } 34 \% \text { a la formación tecnológica, } \\
\text { En un } 42 \% \text { a la formación científica, } \\
\text { En un } 42 \% \text { a la formación ecológica, } \\
\text { En un } 48 \% \text { a la formación social, } \\
\text { En un } 48 \% \text { a lo cultural } \\
\text { En un } 54 \% \text { a lo ético. }\end{array}$ \\
\hline $\begin{array}{l}\text { "Casi } \\
\text { Siempre" }\end{array}$ & $\begin{array}{l}\text { En un } 21 \% \text { a la formación ecológica, } \\
\text { En un } 28 \% \text { a la formación tecnológica, } \\
\text { En un } 30 \% \text { a la formación científica, } \\
\text { En un } 33 \% \text { a la formación patriótica, } \\
\text { En un } 33 \% \text { a lo cultural } \\
\text { En un } 34 \% \text { a la formación espiritual, } \\
\text { En un } 34 \% \text { a lo ético. } \\
\text { En un } 39 \% \text { a la formación social, }\end{array}$ \\
\hline
\end{tabular}

En el caso de los estudiantes, resalta el aspecto ético, evaluado con el porcentaje más alto de todos los ítems, seguido por lo cultural y lo social. Llama la atención la formación patriótica, como el concepto con el porcentaje de calificación más bajo del ejercicio.

Desde esa lógica, se encuentra que, tanto docentes como estudiantes reconocen otorgando los porcentajes más altos a la contribución hacia una formación ética, desde los diversos espacios educativos. En general, se encuentran más coincidencias que contradicciones, entre docentes y estudiantes, en ésta consideración particular y sus diversas opciones, puesto que los porcentajes de respuesta son muy similares en ambos públicos.

Adicionalmente en sus testimonios lo confirman los estratos encuestados, al decir que:

"Con el planteamiento de proyectos sociales y de impacto que puedan dar soluciones a problemas de la comunidad". Testimonio de profesor. 
"Priorizando la solución de problemas y haciendo un cambio en mi. para poder cambiar a la sociedad". Testimonio de estudiante.

Pareciera entonces que, desde las tendencias franciscanas estamos cumpliendo, en tanto señala que:

Sin embargo, estoy convencido de que San Francisco y la tradición franciscana de espiritualidad y búsqueda intelectual han hecho una contribución excepcional al mundo de la educación católica. Ellas tienen una perspectiva especial para ofrecer a los estudiantes, una que llega no solo a sus cerebros sino también a sus corazones, una perspectiva que insiste en que los conceptos agudos de la mente y los afectos profundos del corazón deben convertirse en la sustancia del servicio práctico para las grandes necesidades del mundo. (Perry, 2013, p. 16).

Adicionalmente, en cuanto a lo tecnológico, expresa en Id y enseñad que:

Por otra parte, el avance de los medios de comunicación y, particularmente, la migración de los pueblos de un país o de una región a otra, ponen de relieve el nuevo contexto en el que nos desenvolvemos: la diversidad y la pluralidad cultural, étnica, racial, lingüística, religiosa, social, politica y económica. Un espacio vital con oportunidades y amenazas, posibilidades y limitaciones. (OFM, 2009, p. 10).

En síntesis, puede caracterizarse la Visión institucional, en términos de fortalezas y debilidades en el siguiente cuadro:

\begin{tabular}{|c|c|c|c|}
\hline \multicolumn{4}{|c|}{ CATEGORÍA VISIÓN } \\
\hline \multicolumn{2}{|c|}{ FORTALEZAS } & \multicolumn{2}{|c|}{ DEBILIDADES } \\
\hline PROFESORES & ESTUDIANTES & PROFESORES & ESTUDIANTES \\
\hline $\begin{array}{l}\text { Se muestra una tendencia } \\
\text { relevante de parte de los } \\
\text { docentes, a ver la innovación } \\
\text { pedagógica como uno de los } \\
\text { elementos más relevantes de } \\
\text { la visión de la Universidad. } \\
\text { lgualmente lo relacional tiene } \\
\text { un peso fundamental para el } \\
\text { docente en su labor } \\
\text { académica. } \\
\text { Llama la atención que los } \\
\text { elementos de tipo social y } \\
\text { relacional, tengan un peso tan } \\
\text { significativo en las } \\
\text { evaluaciones de los docentes; } \\
\text { es decir, en general pareciera } \\
\text { que son conscientes de la } \\
\text { importancia de formar } \\
\text { personas humanas y } \\
\text { profesionales. }\end{array}$ & $\begin{array}{l}\text { El estudiante da una } \\
\text { importancia fundamental a lo } \\
\text { relacional y reconoce la } \\
\text { innovación en términos } \\
\text { pedagógicos, por parte del } \\
\text { docente, como un factor muy } \\
\text { significativo. } \\
\text { Los estudiantes parecen ser } \\
\text { reflexivos en torno al ejercicio } \\
\text { de su profesión desde lo } \\
\text { social; es decir, consideran el } \\
\text { hecho que la academia debe } \\
\text { beneficiar a la sociedad con el } \\
\text { conocimiento generado. }\end{array}$ & $\begin{array}{l}\text { Es fundamental que el } \\
\text { elemento científico sea } \\
\text { reconocido con un mayor peso } \\
\text { en los elementos centrales de } \\
\text { la visión, pues no se concibe la } \\
\text { docencia sin la ciencia y } \\
\text { mucho menos sin la } \\
\text { investigación; ambos asuntos } \\
\text { debe afianzarse mucho más. El } \\
\text { docente debe comprender que } \\
\text { su labor implica investigar. }\end{array}$ & $\begin{array}{l}\text { Al igual que sucede en relación a } \\
\text { las observaciones realizadas en } \\
\text { torno a los docentes, es de gran } \\
\text { importancia que el estudiante } \\
\text { reconozca el asunto científico con } \\
\text { un mayor peso, en cuanto a los } \\
\text { elementos de la visión; si bien la } \\
\text { formación del estudiante de } \\
\text { pregrado no hace énfasis en } \\
\text { aspectos de tipo investigativo, es } \\
\text { de vital importancia establecer } \\
\text { estrategias y ejecutar acciones } \\
\text { que faciliten el desarrollo de un } \\
\text { interés mayor en torno a la } \\
\text { investigación y la indagación, en } \\
\text { tanto ello permite que el } \\
\text { estudiante continúe su proceso } \\
\text { formativo más allá del aula. El } \\
\text { hecho de valorar el asunto de la } \\
\text { ciencia con un porcentaje que } \\
\text { podría valorarse como bajo, en } \\
\text { términos de lo que es una } \\
\text { Universidad, implica que se } \\
\text { deben ejercer acciones al } \\
\text { respecto. }\end{array}$ \\
\hline
\end{tabular}

Cuadro 2. Fortalezas y debilidades de la categoría Visión 


\section{Desde las Dimensiones de la pedagogia franciscana.}

En torno a las dimensiones de la pedagogía franciscana, los docentes manifiestan que el elemento:

- Afectividad, se encuentra "siempre" presente en las asignaturas, con un porcentaje del $56 \%$, y "casi siempre" con un $31 \%$.

- El respeto y la espontaneidad está presente "siempre", según la mirada de los docentes, con un $82 \%$; "casi siempre" con un $15 \%$.

- En cuanto al ritmo de aprendizaje, se comprende que este se tiene en cuenta en los procesos de enseñanza "siempre" en un 60\% y "casi siempre" en un $36 \%$.

- Para los docentes el estilo de aprendizaje mediante los procesos; "siempre" para un 51\% y "casi siempre" para un $42 \%$.

En la percepción de los estudiantes en torno a estas mismas dimensiones de la pedagogía, se encuentra que:

- La afectividad está "siempre" presente en los procesos, para un 43\%, y "casi siempre" para un $37 \%$.

- El respeto y la espontaneidad se evidencian "siempre", para un 40\%, y "casi siempre" para un $46 \%$.

- Los ritmos de aprendizaje median los procesos "siempre" para un 43\%; "casi siempre" para un $48 \%$.

- Los estilos de aprendizaje inciden "siempre" en un 48\% y "casi siempre" en un 39\%.

Llama la atención que el respeto y la espontaneidad es el elemento con mayor porcentaje para los docentes; en el caso de los estudiantes es el estilo de aprendizaje.

De otro lado, la afectividad y el estilo de aprendizaje se evalúan de una forma muy similar, en ambos roles docente y estudiante.

En cuanto al respeto y la espontaneidad son los elementos, en los cuales menos coincidencia se encuentra, en las percepciones de los dos roles docente-estudiante. Para el docente este ítem tiene el grado más alto de calificación; no así para el estudiante, quien considera que el respeto está presente "casi siempre", (más no siempre), en los espacios formativos. Para el docente el ritmo de aprendizaje, media y transversaliza las relaciones con los dicentes; en el caso del alumno, la respuesta es "casi siempre", la que obtiene el mayor resultado en este mismo ítem.

En los testimonios se constató la anterior situación, cuando plantean:

Testimonios de profesores:

"Podría considerarse, que ante todo el respeto por el otro, teniendo en cuenta la forma de pensar, las decisiones que toma y la forma de actuar sobre las mismas, ya que de allí parte, el reflejar los demás valores que crean un espíritu bonaventuriano."

"Escuchando y aconsejando a los estudiantes orientándolos hacia un enfoque más humano. Ser amables con ellos y siempre respetar su postura". 
Testimonios de estudiantes:

"Respetando cada miembro de la comunidad con sus diferencias".

"Primero que todo debemos respetar la opinión de los demás, segundo debemos tener en cuenta que cuando trabajamos en equipo que debemos ser claros, para que no solo uno trabaje".

En esa línea, desde las directrices generales de la educación franciscana, se propone:

La persona, tanto en su ser como en su existencia particular se revela como un misterio único e irrepetible, es decir: con una originalidad y libertad dignas de un profundo respeto. (OFM, 2009, p. 24).

En lo referido a, si en las diversas asignaturas/cursos/seminarios que se desarrollan en el proceso de formación profesional se exploran las múltiples facetas de la persona como ser capaz de captar, dar y expresar sentido a la realidad mediante el diálogo Consigo mismo, Con el otro, Con el entorno y Con el trascendente; se encontró en el caso de los docentes:

- En la opción diálogo consigo mismo: "siempre" en un 64\% y "casi siempre" en un $22 \%$.

- En el ítem diálogo con el otro, "siempre" un $75 \%$ y "casi siempre" un $22 \%$.

- En diálogo con el entorno, "siempre" en un 67\% y "casi siempre" en un $22 \%$.

- En la opción del diálogo con el trascendente, "siempre" en un 36\% y "casi siempre" en un $35 \%$.

En cuanto al estrato de estudiantes, arrojó:

- El diálogo consigo mismo "siempre" en un 54\% y "casi siempre" en un 30\%.

- En el diálogo con el otro "siempre" en un $49 \%$ y casi siempre en un $40 \%$.

- En el diálogo con el entorno "siempre" en un $48 \%$ y "casi siempre" en un $45 \%$.

- En el diálogo con el trascendente; "siempre" un 45\% y "casi siempre" un 33\%.

A partir de esto puede afirmarse que el concepto con mayor calificación fue, en el caso de los docentes, el diálogo con el otro; en el caso de los estudiantes el diálogo consigo mismo. Es de resaltar que el ítem con menor calificación de esta pregunta, tanto en el caso de docentes como de estudiantes, fue el diálogo con el trascendente.

Pareciera entonces que como institución franciscana debemos fortalecer ese diálogo con el trascendente y posiblemente debamos iniciar por la autotrascendencia que "exige que sintamos profundamente a los demás. Exige que nos convirtamos en hombres y mujeres compasivos" (OFM, 2009, p. 19).

No obstante, se encuentra que en otras relaciones dialógicas vamos por el camino señalado desde los postulados franciscanos, en tanto: 
Testimonio de profesor;

"Lo principal es desarrollar la formación con una misión de dignidad, y fraternidad entre las personas, capacitándonos con miras a coadyuvar a la solución de los grandes temas y problemas que enfrenta nuestra sociedad".

Testimonio de estudiante;

"Sabiendo que mi formación no solo es un acto que depende de mí, sino de una interacción de compañeros alumnos, docentes pues de una u/otra forma aportan experiencias para mi futuro".

En sintesis, puede caracterizarse las Dimensiones de la pedagogía franciscana, en términos de fortalezas y debilidades en el siguiente cuadro:

\begin{tabular}{|c|c|c|c|}
\hline \multicolumn{4}{|c|}{ CATEGORÍA DIMENSIONES DE LA PEDAGOGÍA FRANCISCANA } \\
\hline \multicolumn{2}{|c|}{ FORTALEZAS } & \multicolumn{2}{|c|}{ DEBILIDADES } \\
\hline PROFESORES & ESTUDIANTES & PROFESORES & ESTUDIANTES \\
\hline $\begin{array}{l}\text { El docente muestra relación con } \\
\text { la filosofía franciscana y su } \\
\text { vivencia en lo cotidiano. Sería } \\
\text { necesario evaluar si igualmente } \\
\text { existe una apropiación teórica. } \\
\text {-Se observa una alta convicción } \\
\text { de los docentes en la necesidad } \\
\text { de formar en la fraternidad, la } \\
\text { alteridad, } \\
\text { La alteridad, la racionalidad, la } \\
\text { fraternidad son elementos } \\
\text { axiológicos que se muestran } \\
\text { como fortalezas de la propuesta } \\
\text { pedagógica franciscana. } \\
\text { El docente reconoce elementos } \\
\text { esenciales en un nivel alto como: } \\
\text { Rigor científico, análisis serio de } \\
\text { la realidad, orientación a la } \\
\text { realidad social, espíritu } \\
\text { investigativo } \\
\text { El docente se auto reconoce } \\
\text { como facilitador de aprendizaje y } \\
\text { creativo, más que un poseedor } \\
\text { del conocimiento absoluto }\end{array}$ & $\begin{array}{l}\text { Los estudiantes consideran como } \\
\text { relevantes en su proceso de } \\
\text { aprendizaje, muchos de los } \\
\text { elementos que constituyen la } \\
\text { "pedagogía franciscana" } \\
\text { La educación franciscana no } \\
\text { privilegia el saber por el saber } \\
\text { mismo, ni ve la ciencia como la } \\
\text { verdad absoluta, ni la razón como } \\
\text { un fin en sí mismo. Son } \\
\text { elementos que contribuyen a la } \\
\text { transformación del ser humano y } \\
\text { de la sociedad, sin dejar de lado } \\
\text { otros elementos igualmente } \\
\text { valiosos como lo afectivo, la } \\
\text { experiencia y lo vivencial. } \\
\text { Hay un reconocimiento unánime } \\
\text { de la intencionalidad humanista, } \\
\text { aspecto relacionado con lo } \\
\text { confesional, con lo católico, con } \\
\text { lo religioso, se asume con } \\
\text { respetuosa y neutral distancia en } \\
\text { los discentes. } \\
\text { El reconocimiento en un nivel } \\
\text { alto, por encontrar en las }\end{array}$ & $\begin{array}{l}\text { Los docentes tienen una visión } \\
\text { positiva de su propia labor, frente } \\
\text { a la aplicación cotidiana de las } \\
\text { dimensiones de la pedagogía; } \\
\text { pero existe una brecha de esta } \\
\text { mirada en relación con las } \\
\text { percepciones que sobre el mismo } \\
\text { asunto tienen los estudiantes. } \\
\text { El docente manifiesta que en el } \\
\text { énfasis formativo la dimensión } \\
\text { patriótica es baja } \\
\text { La dimensión espiritual de los } \\
\text { alumnos y futuros profesionales, } \\
\text { no constituye una prioridad } \\
\text { dentro de las asignaturas } \\
\text { formalmente establecidas, pero } \\
\text { cabe anotar, sí viene siendo } \\
\text { atendida desde espacios alternos } \\
\text { como Pastoral Universitaria }\end{array}$ & $\begin{array}{l}\text { El estudiante no percibe } \\
\text { claramente lo que el docente } \\
\text { da por elemental: la vivencia } \\
\text { de los elementos asociados a } \\
\text { la pedagogía franciscana. }\end{array}$ \\
\hline
\end{tabular}

Cuadro 3. Fortalezas y debilidades de la categoría Dimensiones de la pedagogía franciscana.

\section{Conclusiones.}

Habiendo presentado el detalle cuantitativo y cualitativo frente a este propósito curricular e institucional, el grupo investigador puede afirmar lo siguiente:

El estudio deja abierto y en plena vigencia un espacio para el análisis crítico-propositivo a nivel institucional, luego de caracterizar las percepciones declaradas por los estudiantes y profesores sobre las vivencias/experiencias de la Misión y Visión de la USB-Medellín, lo 
cual contribuye por un lado, a los procesos de autoevaluación permanente con fines de mejoramiento continuo y por otro lado, a la reflexión-acción de dos de los aspectos esenciales del Proyecto Educativo Bonaventuriano (Misión, Visión articulados a las dimensiones de la pedagogía franciscana) y el consecuente aporte para la acreditación de alta calidad.

- Es claro para quienes participaron en la encuesta que los asuntos de corte ético, social, formativo, son de vital importancia para la comunidad académica y que se afirman desde el hacer y sentir del docente y el estudiante.

- Es interesante observar como el asunto ético y el componente social son bien evaluados y admitidos como elementos sustanciales dentro del quehacer académico, tanto para estudiantes como para docentes.

- Se constata con el estudio que existe en general, una vivencia de los elementos filosóficos y de identidad consagrados en la misión y la visión de la Universidad de San Buenaventura; el quehacer cotidiano se impregna de una serie de componetes particulares, y aunque muchos pudieran no conocerlos al detalle, se evidencia la comprensión de su aplicabilidad en la vida diaria de la Institución.

- Llama la atención que el docente, de manera general, parece tener mayor apropiación de los elementos franciscanos que el estudiante. Ello debería entonces facilitar la generación de una transmisión significativa de los valores y las dimensiones de la filosofia franciscana y bonaventuriana en el proceso de enseñanza. Se deduce de lo observado que el docente se apropia de ello, pero todavia hay carencias en la transmisión de esos elementos a los estudiantes, tal vez los alumnos no lo perciben con claridad.

- Es también llamativo que la vivencia de lo evangélico-cristiano, específicamente lo relacionado con la dimensión interior y espiritual no sea tan recurrente en algunas respuestas. Pareciera ser que le dimensión cristiana y su vivencia se observa única y exclusivamente desde el credo religioso, que no siempre es apreciado y compartido por todos. El equipo investigador puede deducir entonces, entre otros, que no se comprende la vivencia de la dimensión cristiana como una serie de valores implicados a lo largo de distintos espacios identitarios pedagógicos y curriculares de la Universidad. Igual sucede con el elemento franciscano.

La propuesta franciscana se constituye en un aporte importante ante al actual crisis de sentido, en la recuperación del sujeto en procura de "asumir nuestra historicidad y la indelegable tarea de transformar, de manera eficiente, nuestro entorno desde lo que somos. Nos pone la tarea perentoria de rescatar al sujeto y potenciarlo desde su realidad, construir para tal fin, las condiciones para la formación de sujetos capaces de problematizar su entorno, de elaborar preguntas y construir respuestas desde el saber propios" (Insuasty Rodriguez, 2013).

Es por ello que se hace fundamental alimentar las reflexiones que sobre los fundamentos filosóficos se desarrollan en la USB Medellín, con miras a la ampliación del campo de conocimiento acerca de asuntos como lo cristiano y lo franciscano, que como cosmovisiones, 
formas de sentir, de pensar y de ver el mundo, entran en diálogo con otras maneras de comprender y vivenciar la realidad del sujeto.

Es clave por ejemplo, ahondar en la percepción en torno a los valores como la fraternidad en tanto que para el grupo de Investigación: "posibilita la fraternidad una convivencia pacífica y una economía solidaría en tanto ella exige una decisión férrea de la voluntad consciente de los colectivos que luchan por una vida digna, lo que implicaría para estos, cambiar las relaciones sociales, politicas, de producción e incluso normativas que rigen dichas relaciones y la forma como se asumen los conflictos que en dichos colectivos se suscitan" (Morales \& Insuasty Rodriguez, 2011).

Es fundamental que se entienda lo cristiano y franciscano desde sus elementos esenciales, puesto la investigación comprueba que en general existe cierta resistencia hacia esas temáticas.

\section{Referencias Bibliográficas.}

Arroyave Giraldo, D. I., \& Carrión Suárez, S. E. (2013). Primera Jornada de trabajo. Propuesta de lineamientos curriculares. Universidad de San Buenaventura Medellín, Medellín.

Arroyave Giraldo, D. I., Cardona Ramírez, C. M., Sánchez Sánchez, L. F., Moreno López, V., Carrión Suárez, S. E., \& Cuervo , J. E. (2015). Vivencias/experiencias de estudiantes y profesores sobre la misión y visión de Universidad de San Buenaventura-Medellín. Medellín.

Borg, M. G. (1998). Secondary school teachers "perceptions of pupils" undesirable behaviours. British Journal of Educational Psychology(68), 67-79.

Cardona, C., Muñoz, D., \& Velasquez, J. (2007). La Paideia Franciscana: una mirada a la expansión humana. Medellin: Universidad de San Buenaventura seccional Medellín.

Hernández, R., Fernández, C., \& Baptista. (2003). Metodología de la investigación (3 ed.). México DF: McGraw-Hill.

Hungler, B., \& Polit, D. (2000). Investigación científica en Ciencias de la Salud (5 ed.). México DF: McGraw-Hill.

Insuasty Rodriguez, A. (2013). Ante la actual crisis de sentido, es necesario "rescatar al sujeto". El Agora USB, 13(2), 8-12.

Morales , A., \& Insuasty Rodriguez, A. (2011). Fraternidad para la vida digna de los Pueblos. El Agora USB, $11(2), 435-454$.

Orden Franciscana Menor - Curia generale dei Frati Minori. (2009). Id y Enseñad. Directrices generales para la Educación Franciscana. Roma, Italia.

Pérez, J. A. (1989). Percepción y Categorización del contexto social. En Y. Seoane, \& A. Rodríguez, Creencias, Actitudes y Valores. Madrid: Alhambra Longmaxn. 
Perry, M. A. (2013). La educación franciscana como un agente de cambio. Cuarta Reunión de los Centros Franciscanos de Educación Superior en América y España. Bogotá.

Universidad de San Buenaventura. (2007). PEB Proyecto educativo Bonaventuriano. Bogotá: Universidad de San Buenaventura.

Universidad de San Buenaventura Medellín. (2012). Plan Institucional 2013-2017. Medellín.

\section{Nota:}

${ }^{1}$ Agradecimiento especial al equipo de trabajo: Carlos Mario Cardona Ramírez vicerrector Académico Universidad de San Buenaventura Medellin, Sandra Elena Carrión Suarez Asistente y Juan Esteban Cuervo facultad de Artes integradas Universidad de San Buenaventura Medellin. 